LA W RENCE LIVERM ORE N A TIO NAL LABORATORY

Modeling Electro-Magnetic Fields from HE Explosions

A. L. Kuhl

May 28, 2013 
This document was prepared as an account of work sponsored by an agency of the United States government. Neither the United States government nor Lawrence Livermore National Security, LLC, nor any of their employees makes any warranty, expressed or implied, or assumes any legal liability or responsibility for the accuracy, completeness, or usefulness of any information, apparatus, product, or process disclosed, or represents that its use would not infringe privately owned rights. Reference herein to any specific commercial product, process, or service by trade name, trademark, manufacturer, or otherwise does not necessarily constitute or imply its endorsement, recommendation, or favoring by the United States government or Lawrence Livermore National Security, LLC. The views and opinions of authors expressed herein do not necessarily state or reflect those of the United States government or Lawrence Livermore National Security, LLC, and shall not be used for advertising or product endorsement purposes.

This work performed under the auspices of the U.S. Department of Energy by Lawrence Livermore National Laboratory under Contract DE-AC52-07NA27344. 


\title{
Modeling Electro-Magnetic Fields from HE Explosions
}

\author{
A. L. Kuhl \\ Lawrence Livermore National Laboratory
}

\begin{abstract}
In this paper we study the electro-magnetic (EM) fields generated by the detonation of high-explosives (HE) charges. We propose that such EM emissions are created when the conductive cloud of the detonation products (DP) gases expands in the earth's magnetic field. Ohm's law is used to predict the currents created by the flow field, while the flow field itself is computed by a high-order Godunov solution of the reactive gas-dynamic equations. Ampere's law and Faraday's law are used to model the magnetic and electric fields, respectively. It is shown that the expansion of the $D P$ cloud creates a magnetic dipole moment. An approximate analytic solution is found; it shows that the magnetic induction field falls off as the inverse cube of distance, and decays as the inverse cube-root of time as the blast wave expands.
\end{abstract}

\section{Introduction}

In this paper we develop models to describe electro-magnetic (EM) effects generated by the detonation of high-explosives charges. As a starting point, we review conductivity measurements made in explosion experiments in a shock tube ${ }^{1}$. A two-meter long steel shock tube, with a rectangular cross-section of $10 \mathrm{~cm} \times 10 \mathrm{~cm}$, was constructed (see Fig. 1). It was mounted on a plastic (macrolon) plate to electrically isolate it from the metal support stand. The charge consisted of a $0.5-\mathrm{g}$ PETN booster, surrounded by a 1-g spherical shell of TNT or 1-g of flake Aluminum powder. A schematic of the charge construction is shown in Fig. 2. Instrumentation consisted of piezo-electric gauges (Kistler 603B) to measure the pressure history of the blast waves, photo-diodes to track the luminous region of the flow, and a conductivity probe (Fig. 3) to measure the conductivity history of the cloud. Figure 4 presents a wave diagram of the explosion. The charge was detonated at $x=20 \mathrm{~cm}$, creating a blast wave that propagated down the tunnel. The explosion cloud followed behind the shock; its trajectory was mapped with the photo-diode records. Blast reflection from the end wall $(x=180 \mathrm{~cm})$ reheated the cloud as indicated by the luminous intensity scale shown in Fig. 4. Figure 5 presents the conductivity history measured at $x=124 \mathrm{~cm}$. Conductivity peaked at $\sim 0.023$ Siemens per meter and then decayed to zero in $\sim 4 \mathrm{~ms}$ due to electron recombination processes. The reflected shock reheated the cloud causing a second pulse of conductivity. Photo-diode measurements showed that the conductive region coincided with the luminous cloud.

We have studied conductivity effects in unconfined clouds by performing numerical simulations with our AMR code ${ }^{7}$. The blast wave from a 1-kg spherical TNT charge is presented

\footnotetext{
${ }^{1}$ A. L. Kuhl, Conductivity Histories Measured in Shock-Dispersed-Fuel Explosion Clouds, Lawrence Livermore National Laboratory, LLNL-TR-427483, 2010, 18 pp.
} 
in Fig. 6. When the detonation wave reaches the charge boundary, it accelerates the detonation products (DP) - air interface to $6.5 \mathrm{~km} / \mathrm{s}$. This interface acts as a spherical piston, driving a strong shock into the surrounding air. Peak air temperature in this thin spherical shell starts at $10,000 \mathrm{~K}$ then decays due to adiabatic expansion. Such temperatures are large enough to create significant ionization in the air, so the air shell becomes conductive. Details of the shock-heated air region are shown in Fig. 7-11. Evidently the conductive region is very thin-approximately $1 \mathrm{~mm} / \mathrm{kg}^{1.3}$ at this time $\left(2.5 \mu \mathrm{s} / \mathrm{kg}^{1 / 3}\right)$.

According to Ohm's Law, this moving conductive shell creates an azimuthal current, which then induces a magnetic dipole moment according to Ampere's Law. We call this the Boronin Effect, in honor of A. P. Boronin who explored various mechanisms of generating electromagnetic effects in HE explosions ${ }^{2}$. We now derive a model of the EM fields generated by such magnetic dipoles, based on the Maxwell equations.

\section{Formulation}

Consider the explosion of a spherical HE charge in the earth's magnetic field. We search for models of electromagnetic waves created such explosions. We start our analysis with Ohm's Law (A1):

$$
\mathbf{J}=\sigma(\mathbf{E}+\mathbf{v} \times \mathbf{B})
$$

where $\mathbf{J}$ denotes current, $\mathbf{E}$ and $\mathbf{B}$ the electric and magnetic field vectors, $\mathbf{v}$ the gas velocity, and $\sigma$ is the gas conductivity. We assume a constant local magnetic induction field $\mathbf{B}_{0}=B_{0} \mathbf{i}_{z}$ devoid of local electric fields $\left(\mathbf{E}_{0}=0\right)$. Then Ohm's Law reduces to:

$$
\mathbf{J}_{\theta}=\sigma \mathbf{v} \times \mathbf{B}_{0}
$$

Expressing this for the spherical explosion field of Fig. 1, one finds:

$$
\mathbf{J}_{\theta}(r, t)=\sigma(r, t) u_{r}(r, t) B_{0} \sin \phi \mathbf{i}_{\theta}
$$

The geometry of this effect is shown in Fig. 12. Note that the current is zero at $\phi=0$ ( $z$ axis).

\section{Approximate Solution for a Spherical TNT explosion}

Appealing to the Biot-Savart Law ${ }^{3}$ one finds the relations:

$$
\mathbf{B}(\mathbf{x}, t)=\frac{\mu_{0}}{4 \pi} \int \mathbf{J}_{\theta}(r, t) \times \frac{\mathbf{x}-\mathbf{x}^{\prime}}{\left|\mathbf{x}-\mathbf{x}^{\prime}\right|^{3}} d^{3} x^{\prime}
$$

\footnotetext{
${ }^{2}$ A. P. Boronin, V. N. Kapinos, S. A. Krenev, V. N. Mineev, Physical mechanism of electromagnetic field generation during the explosion of condensed explosive charges: survey of literature, Combustion, Explosion \& Shock Waves 26 (5) (1990) 597-602.

${ }^{3}$ John D. Jackson, Classical Electrodynamics, $3^{\text {rd }}$ Edition, John Wiley, New York (1999) 808 p (vid. esp. § 5.5 Vector Potential and Magnetic Induction for a Circular Current Loop, pp. 181-188 and Eq. 5.41)
} 


\section{A. L. Kuhl}

$$
\mathbf{E}(\mathbf{x}, t)=\frac{1}{4 \pi} \int \dot{\mathbf{B}}(r, t) \times \frac{\mathbf{x}-\mathbf{x}^{\prime}}{\left|\mathbf{x}-\mathbf{x}^{\prime}\right|^{3}} d^{3} x^{\prime}
$$

They show that current $\mathbf{J}_{\theta}$ creates a magnetic induction field $\mathbf{B}(\mathbf{x}, t)$ whose temporal variation creates an electric field $\mathbf{E}(\mathbf{x}, t)$. Approximating this current as current $J_{\theta}(a, t)$ concentrated in a closed circular loop of radius $a$, the magnetic induction field in the far field $(r>>a)$ becomes:

$$
\begin{aligned}
& B_{r}(r, \phi, t)=\frac{\mu_{0}}{2 \pi}\left[J_{\theta}(a, t) \pi a^{2}\right] \frac{\cos \phi}{r^{3}} \\
& B_{\phi}(r, \phi, t)=\frac{\mu_{0}}{2 \pi}\left[J_{\theta}(a, t) \pi a^{2}\right] \frac{\sin \phi}{r^{3}}
\end{aligned}
$$

The corresponding electric field is:

$$
\begin{aligned}
& E_{r}(r, \phi, t)=\frac{\mu_{0}}{4 \pi a}\left[\dot{J}_{\theta}(a, t) \pi a^{2}\right] \frac{\cos \phi}{r^{3}} \\
& E_{\phi}(r, \phi, t)=\frac{\mu_{0}}{4 \pi a}\left[\dot{J}_{\theta}(a, t) \pi a^{2}\right] \frac{\sin \phi}{r^{3}}
\end{aligned}
$$

This shows that azimuthal current $\mathbf{J}_{\theta}$ induces both magnetic and electric fields. It is useful to express this as specific current (i.e., current per unit magnetic field):

$$
\sigma u_{r}=\left|\mathbf{J}_{\theta} / \mathbf{B}_{0}\right|
$$

The peak specific current computed from the numerical simulation shown in Fig. 6, is plotted as a function of scaled radius and time in Figs. 13 and 14. By fitting the numerical solution, one finds that the peak specific current decays in distance and time as the blast wave expands:

$$
\begin{aligned}
& \left|\sigma \cdot u_{r}\right|_{s}=f_{1}\left(r_{s}\right)=442 / r_{s}^{2} \\
& \left|\sigma \cdot u_{r}\right|_{s}=f_{2}\left(t_{s}\right)=-8+22.6 / t_{s}^{0.277}
\end{aligned}
$$

where $\left[r_{s}\right]=\mathrm{cm} / \mathrm{kg}^{1 / 3}$ and $\left[t_{s}\right]=\mu s / \mathrm{kg}^{1 / 3}$. The corresponding decay rate for the specific current is given by:

$$
\frac{d}{d t}\left|\sigma \cdot u_{r}\right|_{s}=\dot{f}_{2}\left(t_{s}\right)=-6.26 / t_{s}^{1.277}
$$

which is related to $\dot{\mathbf{B}}$. The above relations lie on the $r$ - $t$ locus defined by:

$$
t_{s}=-5.28+0.48 \cdot r_{s}^{1.45} \quad \text { or } \quad r_{s}=5.268+0.791 \cdot t_{s}^{0.855}
$$


Utilizing the fit (12) in the relations (6-7) one finds

$$
\begin{aligned}
& B_{r}(r, \phi, t)=11.3 \frac{\mu_{0} B_{0}}{a} \sin \phi \cos \phi\left(\frac{a}{r}\right)^{3} \frac{1}{t^{0.277}} \\
& B_{\phi}(r, \phi, t)=11.3 \frac{\mu_{0} B_{0}}{a} \sin ^{2} \phi\left(\frac{a}{r}\right)^{3} \frac{1}{t^{0.277}}
\end{aligned}
$$

This represents the approximate analytic solution for the magnetic induction field generated by the expanding detonation products; it falls off with distance as $1 / r^{3}$ and decays as $1 / t^{0.277}$ in time. The magnetic induction field is directional, with a $\sin \phi$ dependence on angle.

\section{Magnetic Induction Field Created by J}

The above analytic solution was based on the approximation that the current could be represented as concentrated in a closed circular loop at radius $a$. But the numerical solution shows that the specific current has a spatial profile with a finite width of $\sim 1 \mathrm{~mm} / \mathrm{kg}^{1 / 3}$ (see Fig. 1f). Also instabilities develop on the DP-air interface, causing $\mathbf{J}_{\theta}$ to become three-dimensional. The interface region transitions to a turbulent mixing region (Fig. 15a) and the fireball becomes a turbulent combustion cloud (Fig. 15b,c). In order to handle the more general case of threedimensional currents: $\mathbf{J}(\mathbf{x}, t)$, we reformulate this problem in terms of partial differential equations, amenable to solution by standard numerical methods for Poisson equations.

We start with the Ampere-Maxwell Law (A2), which relates the magnetic induction field $\mathbf{B}$ to current $\mathbf{J}$ :

$$
\nabla \times \mathbf{B}-\frac{1}{c^{2}} \frac{\partial E}{\partial t}=\mu_{0} \mathbf{J}
$$

Assuming a quasi-static electric field approximation (i.e., $\partial \mathbf{E} / \partial t=0$ ), then the curl of $\mathbf{B}$ is proportional to $\mathbf{J}$ according to

$$
\nabla \times \mathbf{B}=\mu_{0} \mathbf{J}
$$

We also assume that the magnetic induction field obeys Gauss's Law (A4), so

$$
\nabla \cdot \mathbf{B}=0
$$

Since $\mathbf{B}$ is divergence free, it can be related to the curl of the vector potential $\mathbf{b}$ according to

$$
\mathbf{B}(\mathbf{x})=\nabla \times \mathbf{b}(\mathbf{x})
$$

Then (18) becomes 


$$
\nabla \times \nabla \times \mathbf{b}=\mu_{0} \mathbf{J}
$$

Using the vector identity $\nabla \times \nabla \times \mathbf{A}=\nabla(\nabla \cdot \mathbf{A})-\nabla^{2} \mathbf{A}$, equation (21) can be re-written as:

$$
\nabla(\nabla \cdot \mathbf{b})-\nabla^{2} \mathbf{b}=\mu_{0} \mathbf{J}
$$

We choose Coulomb gauge $\mathbf{b}$, such that $\nabla \cdot \mathbf{b}=0$; then (22) reduces to

$$
\begin{aligned}
& \nabla^{2} \mathbf{b}=-\mu_{0} \mathbf{J}_{\theta} \\
& \nabla \cdot \mathbf{b}=0
\end{aligned}
$$

Thus, the problem reduces to solving the vector Poisson equation (23) for a divergence-free vector potential $\mathbf{b}$. Then the magnetic induction field is calculated from the curl of $\mathbf{b}$ :

$$
\mathbf{B}(\mathbf{x}, t)=\nabla \times \mathbf{b}
$$

Since the current $\mathbf{J}(r, t)$ decays with time as the blast wave expands, the corresponding magnetic field $\mathbf{B}(\mathbf{x}, t)$ will also decay with time. One can evaluate its time derivative from finite differences: $\partial \mathbf{B} / \partial t \equiv \dot{\mathbf{B}}^{n} \cong\left(\mathbf{B}^{n+1}-\mathbf{B}^{n-1}\right) / 2 \Delta t$.

\section{Electric Field Induced by $\dot{B}$}

Next we appeal to Faraday’s Law (A3):

$$
\nabla \times \mathbf{E}+\frac{\partial \mathbf{B}}{\partial t}=0
$$

which relates the electric field to the time variation of the magnetic induction field. Since its time variation can be evaluated as $\dot{\mathbf{B}}$, the above reduces to:

$$
\nabla \times \mathbf{E}=-\dot{\mathbf{B}}
$$

Assume the Darwin limit of Maxwell's equations ${ }^{4,5,6}$; then $\mathbf{E}$ is related to be the curl of a vector potential e:

$$
\mathbf{E}(\mathbf{x})=\nabla \times \mathbf{e}(\mathbf{x})
$$

\footnotetext{
${ }^{4}$ C. G. Darwin, Phil. Mag. (1920) 39, 537

${ }^{5}$ D. W. Hewett, D. J. Larson, S. Doss, Solution of the simultaneous partial differential equations using dynamic ADI: Solution of the streamlined Darwin field equations, J. Comp Phys. 101(1), 1992, pp. 11-24.

${ }^{6}$ C. W. Nielson, H. R. Lewis, Particle-code models in the non-radiative limit, Methods of Computational Physics 16, 1976, p. 367-387.
} 
and (27) becomes

$$
\nabla \times \nabla \times \mathbf{e}=-\dot{\mathbf{B}}
$$

Again applying the vector identity $\nabla \times \nabla \times \mathbf{A}=\nabla(\nabla \cdot \mathbf{A})-\nabla^{2} \mathbf{A}$, the above becomes

$$
\nabla(\nabla \cdot \mathbf{e})-\nabla^{2} \mathbf{e}=-\dot{\mathbf{B}}
$$

Assume a gauge transformation such that $\nabla \cdot \mathbf{e}=0$; then the above reduces to:

$$
\begin{aligned}
\nabla^{2} \mathbf{e} & =\dot{\mathbf{B}} \\
\nabla \cdot \mathbf{e} & =0
\end{aligned}
$$

Thus, the problem reduces to solving the vector Poisson equation (30) for a divergence-free vector potential e. The electric field is then computed from the curl operator: $\mathbf{E}(\mathbf{x}, t)=\nabla \times \mathbf{e}$. The electric field will decay with time because $\mathbf{B}$ decays with time.

\section{Model}

The flow field is modeled by the multi-component conservation laws of gasdynamics ${ }^{7}$ :

Mass:

$$
\partial_{t} \rho+\nabla \cdot(\rho \mathbf{u})=0
$$

Momentum:

$$
\partial_{t} \rho \mathbf{u}+\nabla \cdot(\rho \mathbf{u u}+p)=0
$$

Total Energy: $\quad \partial_{t} \rho E+\nabla \cdot(\rho \mathbf{u} E+p \mathbf{u})=0 \quad$ with $E \equiv u(T)+\mathbf{u} \cdot \mathbf{u} / 2$

Components: $\quad \partial_{t} \rho Y_{k}+\nabla \cdot \rho Y_{k} \mathbf{u}=\dot{\omega}_{k} \quad$ with $k=F, A, P$

EOS:

$$
u_{k}(T)=a_{k} T^{2}+b_{k} T+c_{k} \quad \text { and } \quad p_{k}=\rho_{k} R_{k} T_{k}
$$

These equations are integrated in time with a high-order Godunov scheme. The solution is computed on adaptive grid patches that follow discontinuities and reaction zones, and captures all energy-bearing turbulence scales (Fig. 15.) The solution is sampled every time step to determine the conductivity $\sigma(\mathbf{x}, t)$ and velocity $\mathbf{u}(\mathbf{x}, t)$, which are used to construct the current in each cell.

The electromagnetic fields are modeled by the Maxwell equations described in $\S 4$ and $\S 5$. We summarize them as follows:

\footnotetext{
${ }^{7}$ A. L. Kuhl, J. B. Bell, V. E. Beckner, H. Reichenbach, Gasdynamic model of turbulent combustion in TNT explosions, Proceedings of the Combustion Institute 33 (2011) pp. 2177-2185.
} 


\section{A. L. Kuhl}

Current:

$$
\mathbf{J}(\mathbf{x}, t)=\sigma(\mathbf{x}, t) \mathbf{u}(\mathbf{x}, t) \times \mathbf{B}_{0}
$$

Magnetic Potential:

$$
\nabla^{2} \mathbf{b}=-\mu_{0} \mathbf{J} \quad \text { with } \quad \nabla \cdot \mathbf{b}=0
$$

Magnetic Induction:

$$
\mathbf{B}(\mathbf{x}, t)=\nabla \times \mathbf{b}(\mathbf{x}, t)
$$

Time Derivative:

$$
\dot{\mathbf{B}}(\mathbf{x}, t) \cong\left(\mathbf{B}^{n+1}-\mathbf{B}^{n-1}\right) / 2 \Delta t
$$

Electric Potential:

$$
\nabla^{2} \mathbf{e}=\dot{\mathbf{B}} \quad \text { with } \quad \nabla \cdot \mathbf{e}=0
$$

Electric Field:

$$
\mathbf{E}(\mathbf{x}, t)=\nabla \times \mathbf{e}(\mathbf{x}, t)
$$

The magnetic and electric potentials are solved using a predictor-corrector method ${ }^{89}$. In the predictor step, the Poisson equations (38a) and (41a) are integrated with multi-grid elliptic solvers on the same adaptive grid patches used for the flow solver. In the corrector step, these preliminary values are projected onto divergence-free vector spaces. The corrected solution then satisfies $(38 \mathrm{a}, \mathrm{b})$ and $(41 \mathrm{a}, \mathrm{b})$. The magnetic and electric fields are computed with a finite difference curl operator of their respective potentials. The time derivative (40) is computed by a second-order finite-difference operator.

\section{Summary}

A model is proposed to compute the electromagnetic emissions generated during explosion of HE charges. It is based on the fundamental assumption:

EM emissions are created by a conductive (detonation products) cloud moving through the earth's magnetic field.

Ohm's law is used to calculate the currents created by the flow, while the flow field itself is predicted by a high-order Godunov solution of the multi-component conservation laws of reactive gas-dynamics. Ampere's law and Faraday's law are used to model the magnetic induction fields and electric fields induced by the current. At early times, an approximate analytic solution was found ${ }^{3}$ for the magnetic induction field:

$$
|B| \sim B_{0} \sin \phi\left(\frac{a}{r}\right)^{3} \frac{1}{t^{0.277}}
$$

It takes the form of magnetic dipole that is proportional to the earth's local magnetic field strength. It falls off with distance as $1 / r^{3}$ and decays in time as $1 / t^{0.3}$ due to the adiabatic cooling of the ionized air shell as the blast wave expands.

\footnotetext{
${ }^{8}$ J. B. Bell, D. L. Marcus A second-order projection method for variable density flows, J. Comput. Phys. 101 (1992) pp. 334-348.

${ }^{9}$ A. S. Almgren, J. B. Bell, P. Colella,L. H. Howell, M. L. Welcome, A conservative adaptive projection method for the variable density incompressible Navier-Stokes equations, J. Comput. Phys. 142, (1998) 1-46.
} 


\section{Conclusions}

The Model equations are general. At early times, they can simulate the Boronin effect resulting from the expansion of the shock-heated air shell. At later times, they should predict the EM fields created by the combustion patches embedded in the turbulent velocity field.

The next step is to write predictor-corrector algorithms to solve the EM Model equations on the adaptive grid patches in our AMR combustion code. Then run numerical simulations of idealized field test experiments (e.g., 1-kg charges of TNT) and compare with data to ascertain the validity of the EM Model at both early times (Boronin mechanism) and late times (combustion mechanism).

\section{Acknowledgements}

This work performed under the auspices of the U.S. Department of Energy by Lawrence Livermore National Laboratory under Contract DE-AC52-07NA27344.

\section{References}

[1] A. L. Kuhl, Conductivity Histories Measured in Shock-Dispersed-Fuel Explosion Clouds, Lawrence Livermore National Laboratory, LLNL-TR-427483, 2010, 18 pp.

[2] A. P. Boronin, V. N. Kapinos, S. A. Krenev, V. N. Mineev, Physical mechanism of electromagnetic field generation during the explosion of condensed explosive charges: survey of literature, Combustion, Explosion \& Shock Waves 26 (5) (1990) 597-602.

[3] John D. Jackson, Classical Electrodynamics, $3^{\text {rd }}$ Edition, John Wiley, New York (1999) 808 p (vid. esp. § 5.5 Vector Potential and Magnetic Induction for a Circular Current Loop, pp. 181-188 and Eq. 5.41)

[4] C. G. Darwin, Phil. Mag. (1920) 39, 537

[5] D. W. Hewett, D. J. Larson, S. Doss, Solution of the simultaneous partial differential equations using dynamic ADI: Solution of the streamlined Darwin field equations, J. Comp Phys. 101(1), 1992, pp. 11-24.

[6] C. W. Nielson, H. R. Lewis, Particle-code models in the non-radiative limit, Methods of Computational Physics 16, 1976, p. 367-387.

[7] A. L. Kuhl, J. B. Bell, V. E. Beckner, H. Reichenbach, Gasdynamic model of turbulent combustion in TNT explosions, Proceedings of the Combustion Institute 33 (2011) pp. 21772185.

[8] J. B. Bell, D. L. Marcus A second-order projection method for variable density flows, J. Comput. Phys. 101 (1992) pp. 334-348.

[9] A. S. Almgren, J. B. Bell, P. Colella, L. H. Howell, M. L. Welcome, A conservative adaptive projection method for the variable density incompressible Navier-Stokes equations, $J$.

Comput. Phys. 142, (1998) 1-46. 


\section{A. L. Kuhl}

\section{Appendix A: Maxwell's Equations}

Ohm's Law:

$$
\mathbf{J}=\sigma(\mathbf{E}+\mathbf{v} \times \mathbf{B})
$$

Ampere-Maxwell Law: $\quad \nabla \times \mathbf{B}-\frac{1}{c^{2}} \frac{\partial E}{\partial t}=\mu_{0} \mathbf{J}$

Faraday's Law: $\quad \nabla \times \mathbf{E}+\frac{\partial \mathbf{B}}{\partial t}=0$

Gauss's Law (for $\mathbf{E}): \quad \quad \nabla \cdot \mathbf{E}=\rho / \varepsilon_{0}$

Gauss's Law (for $\mathbf{B}$ ): $\quad \nabla \cdot \mathbf{B}=0$

Charge Conservation: $\quad \frac{\partial \rho}{\partial t}+\nabla \cdot \mathbf{J}=0$

where $\mathbf{E} \equiv$ electric field and $\mathbf{D} \equiv$ electric displacement $=\varepsilon_{0} \mathbf{E} ; \mathbf{B} \equiv$ magnetic induction field and $\mathbf{H} \equiv$ magnetic field $=\mathbf{B} / \mu_{0} ; \rho \equiv$ charge density,$\varepsilon_{0}=, \mu_{0}=$ and $c \equiv$ speed of light $=\left(\mu_{0} \varepsilon_{0}\right)^{-1 / 2}$. In the Darwin Model $(1920)^{4}$, the electric field may be decomposed into curl-free and divergence-free components satisfying the relations:
- Irrotational $\mathbf{E}_{\Delta}$ :

$$
\nabla \cdot \mathbf{E}_{\Delta}=\rho / \varepsilon_{0}
$$

$$
\nabla \times \mathbf{E}_{\Delta}=0
$$
- Rotational $\mathbf{E}_{\omega}: \quad \nabla \times \mathbf{E}_{\omega}+\frac{\partial \mathbf{B}}{\partial t}=0$
$\nabla \cdot \mathbf{E}_{\omega}=0$

while Ampere's Law becomes

- Rotational $\mathbf{B}: \quad \nabla \times \mathbf{B}-\frac{1}{c^{2}} \frac{\partial \mathbf{E}_{\Delta}}{\partial t}=\mu_{0} \mathbf{J} \quad \nabla \cdot \mathbf{B}=0$

Note that this only involves the irrotational component of the electric field $\mathbf{E}_{\Delta}$.

\footnotetext{
${ }^{10}$ or $\nabla \times \mathbf{H}-\partial \mathbf{D} / \partial t=\mathbf{J}$

${ }^{11}$ or $\nabla \cdot \mathbf{D}=\rho$
} 


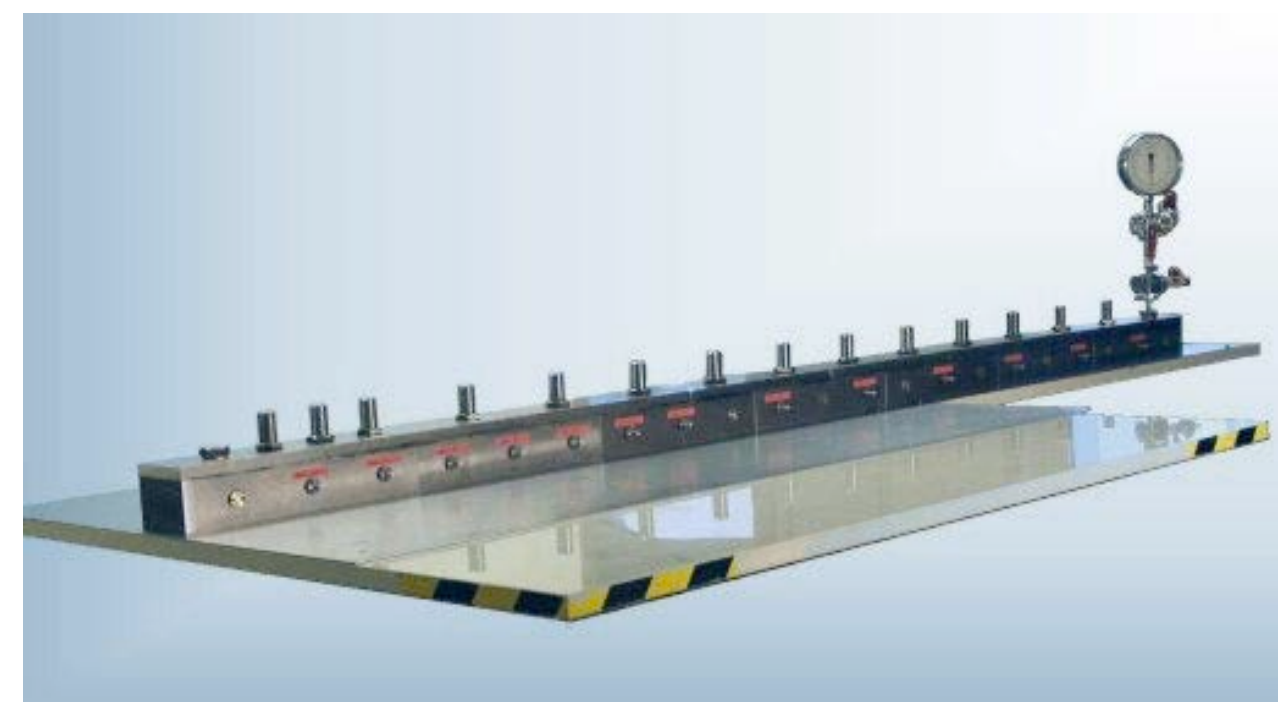

Figure 1. Photograph of the 1-m long tunnel model.

(a) Booster

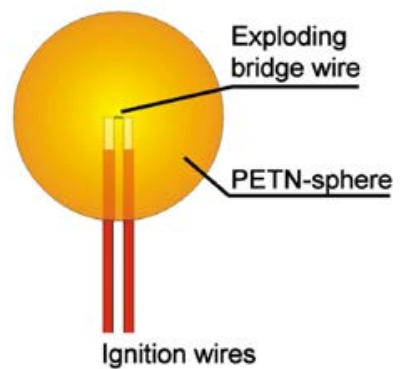

(b) TNT charge

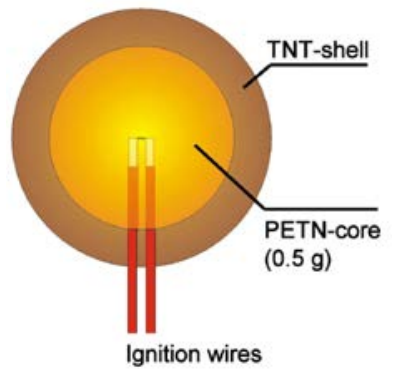

(c) Al-SDF charge

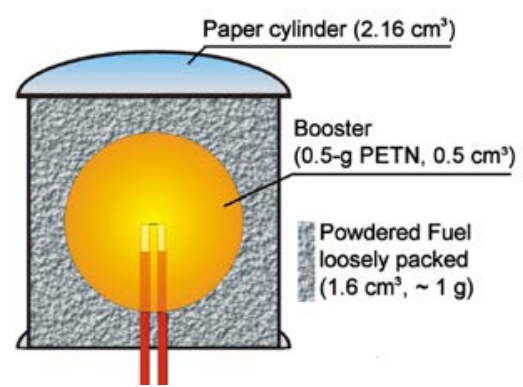

Figure 2. Charge construction: (a) 0.5-g PETN booster charge; (b) composite charge (0.5-g PETN booster + 1-g TNT shell); (c) Al-SDF charge (0.5-g PETN booster + 1-g Aluminum).

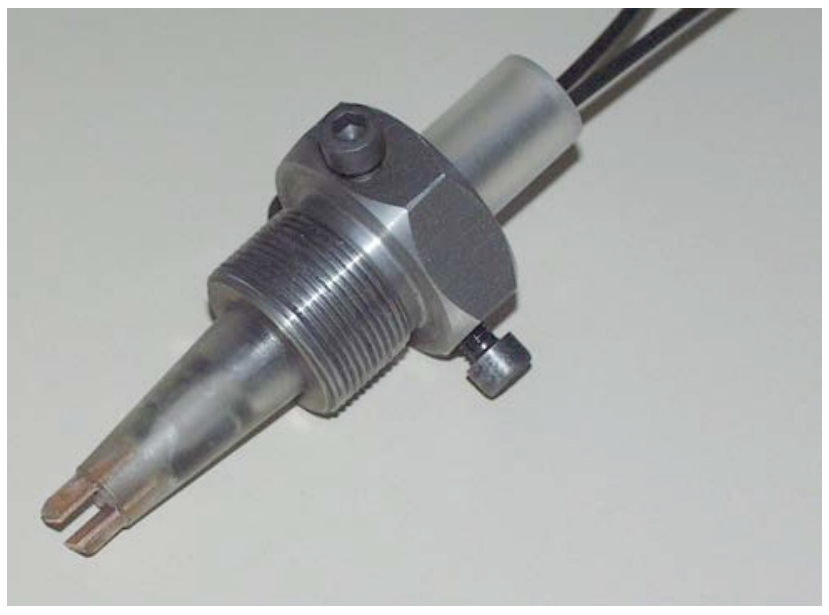

Figure 3. Photograph of the in-flow conductivity sensor. 


\section{A. L. Kuhl}

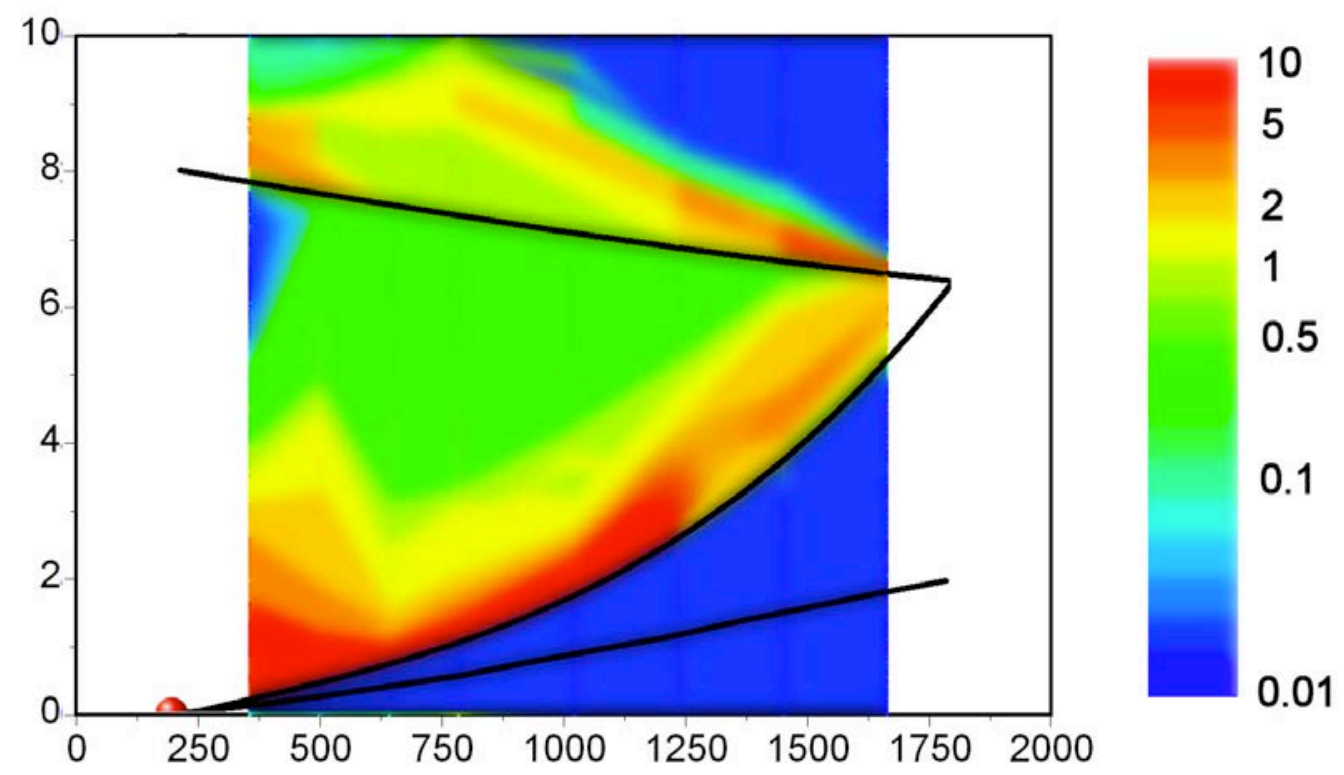

Figure 4. Approximate wave diagram for the conductive region evolving from the detonation of an Al-SDF charge.

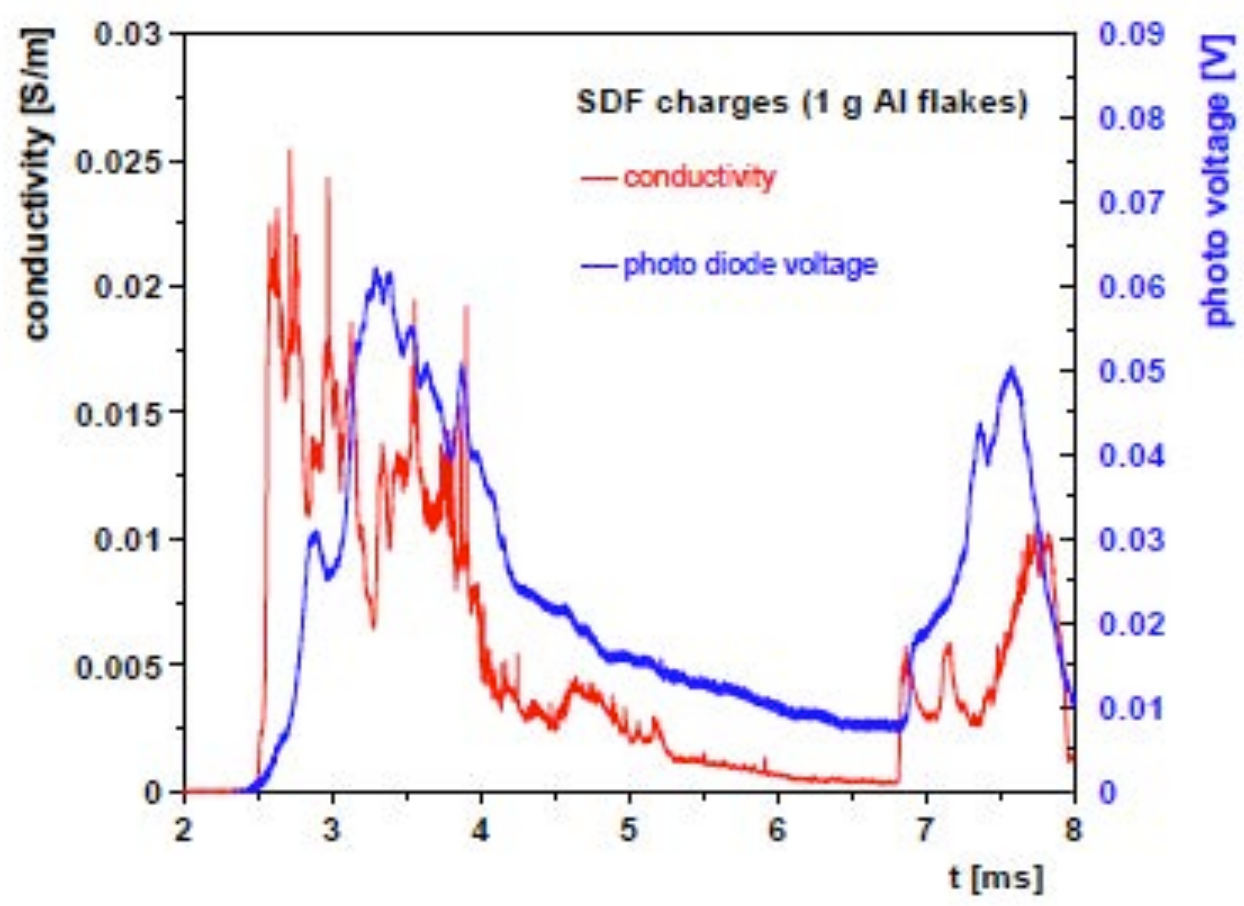

Figure 5. Comparison of the time history of the conductivity (red) and the photo-diode voltage (blue) at $\mathrm{x}=123.6 \mathrm{~cm}$. 
(a) pressure

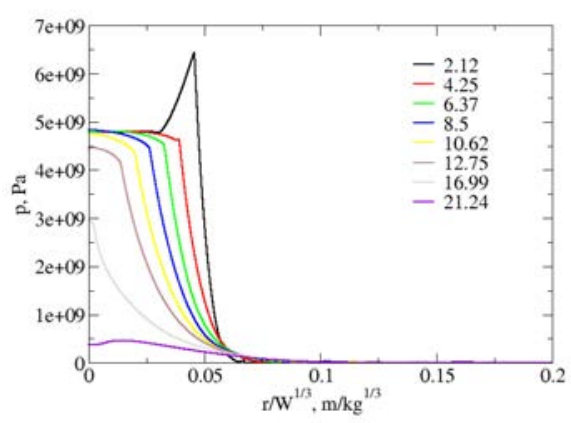

(b) density

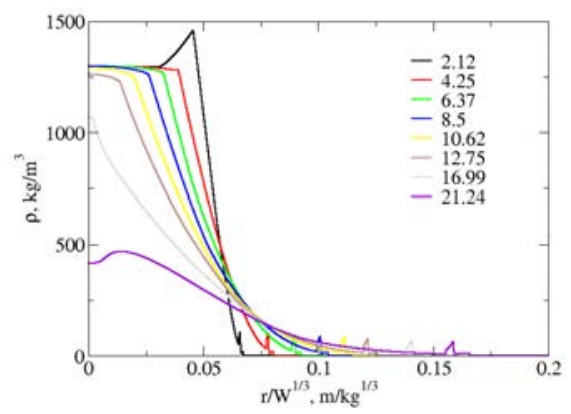

(c) temperature

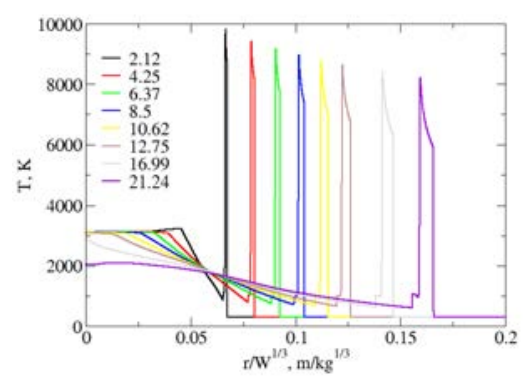

(d) radial velocity

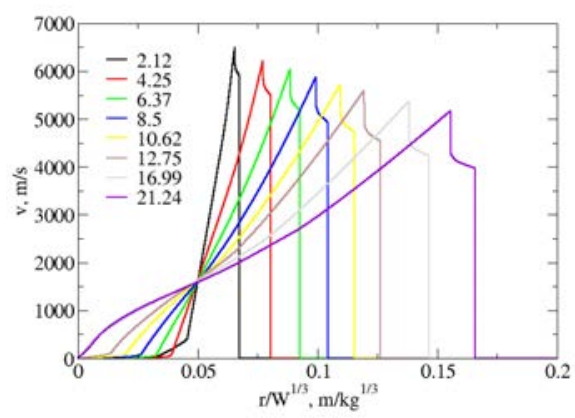

(e) conductivity

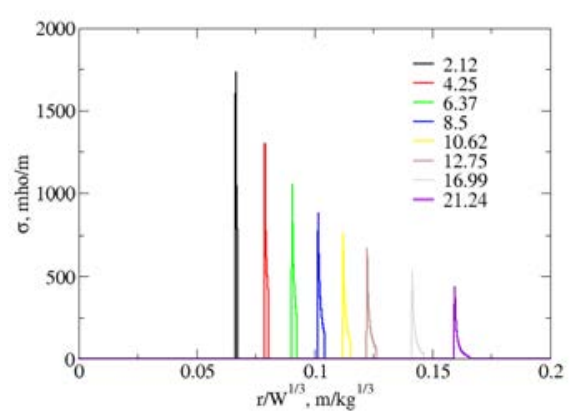

(f) specific current: $\sigma \cdot \mathbf{u}_{r}$

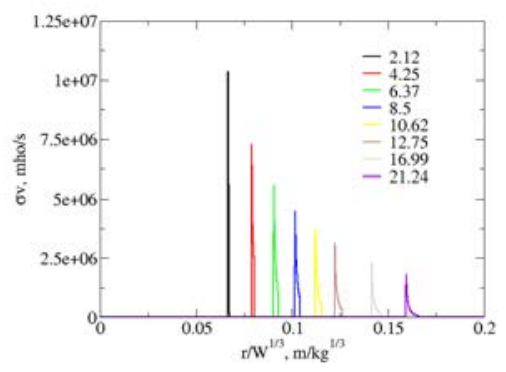

Figure 6. Formation of a blast wave from a 1-kg spherical TNT charge (time legend in $\mu s$ ). 


\section{A. L. Kuhl}

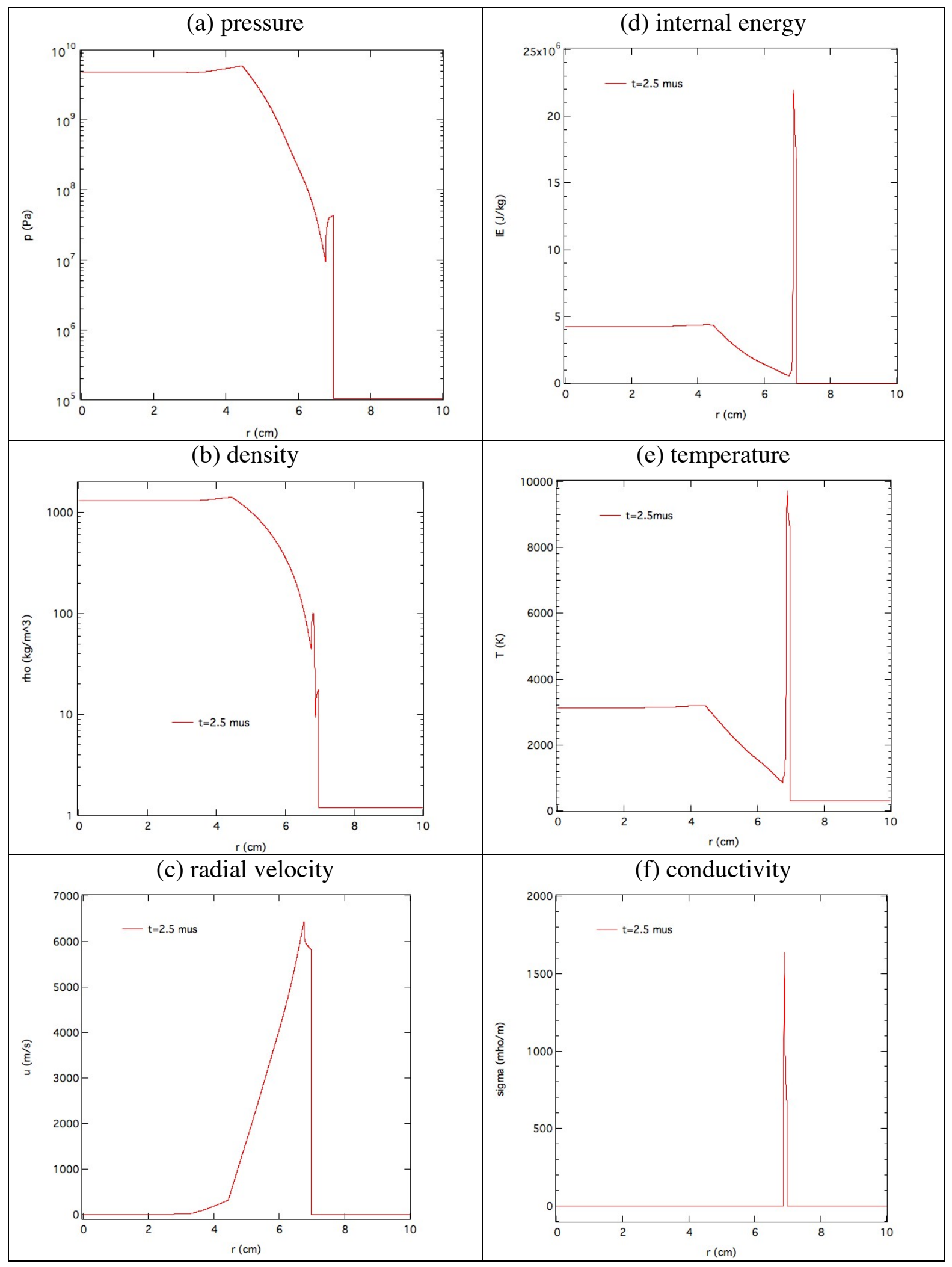

Figure 7. Blast wave flow field from 1-kg TNT explosion at 2.5 microseconds (initial conditions corresponded to a self-similar Chapman-Jouguet detonation wave structure). 
EM Fields from HE Explosions

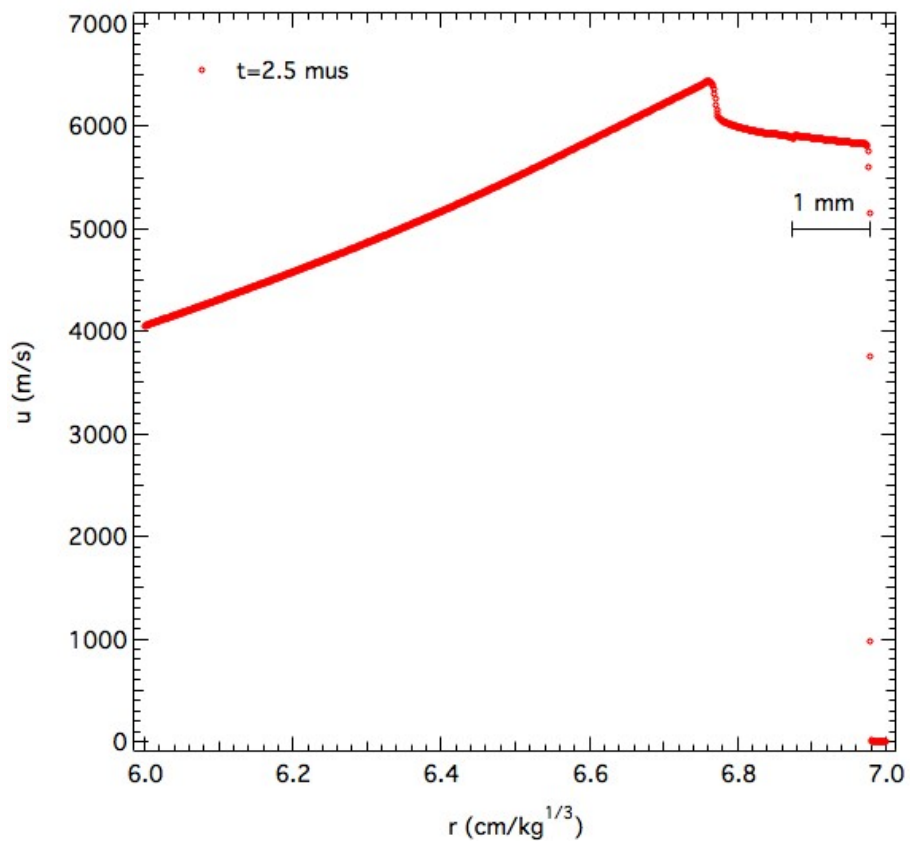

Figure 8. Radial velocity distribution in the shock-heated air layer of a 1-kg TNT explosion at $2.5 \mu \mathrm{s}$.

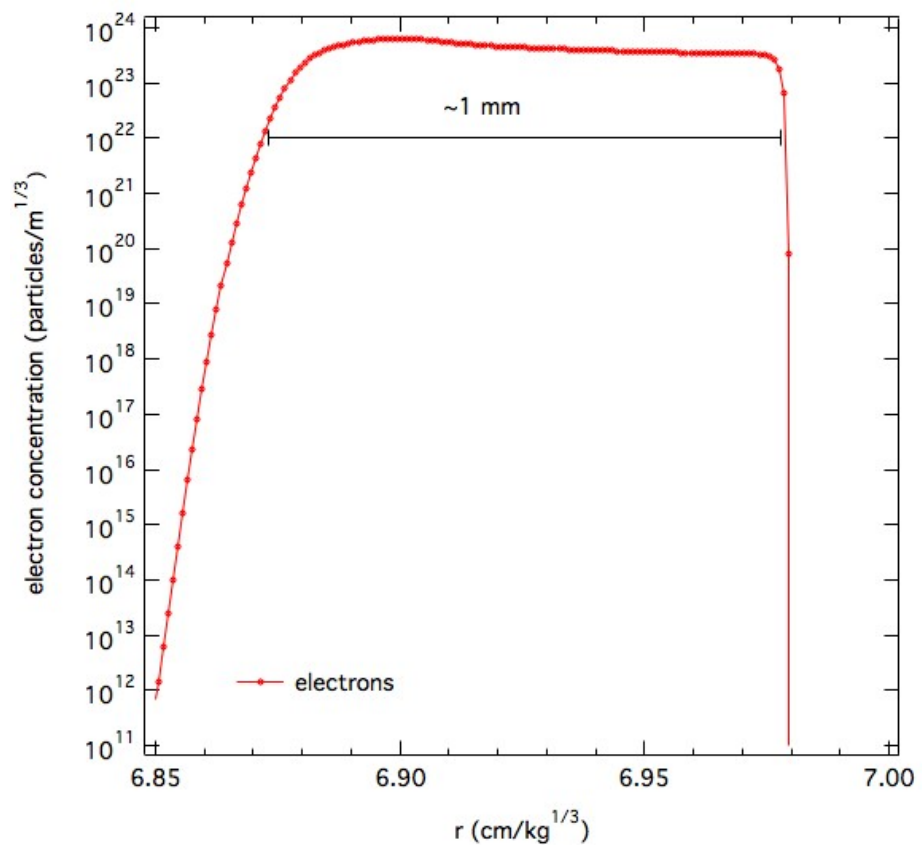

Figure 9. Electron concentration profile in the shock-heated air layer of a 1-kg TNT explosion at $2.5 \mu s$. 


\section{A. L. Kuhl}

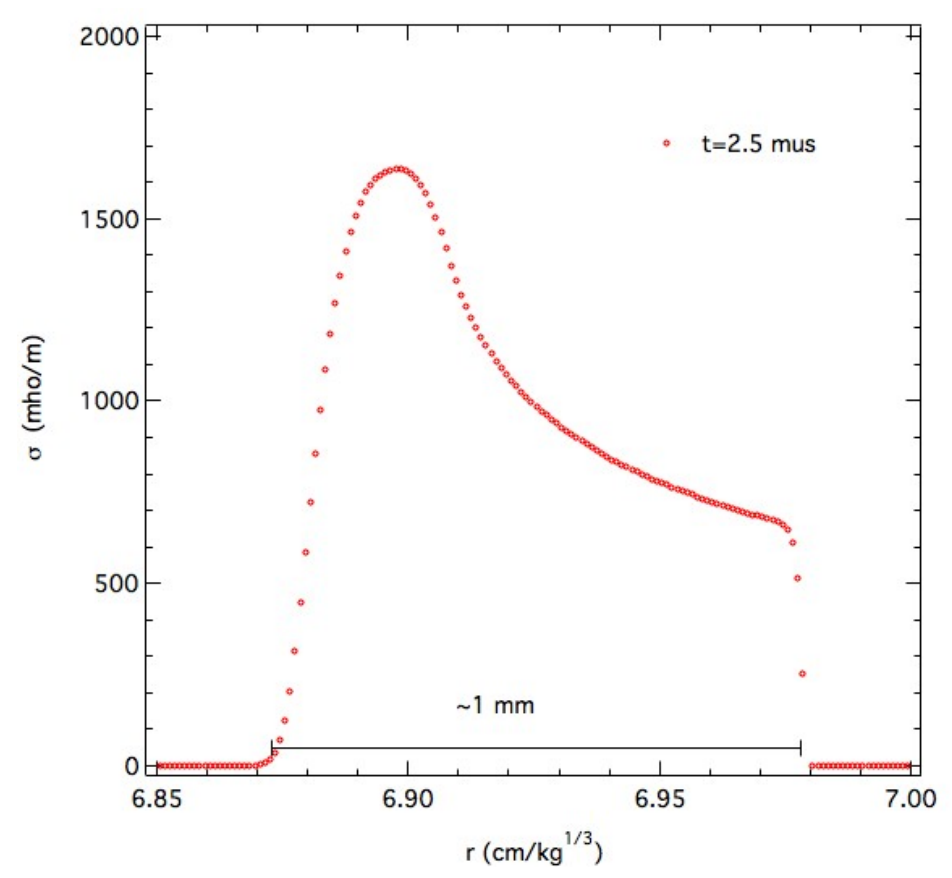

Figure 10. Conductivity distribution in the shock-heated air layer of a 1-kg TNT explosion at $2.5 \mu \mathrm{s}$

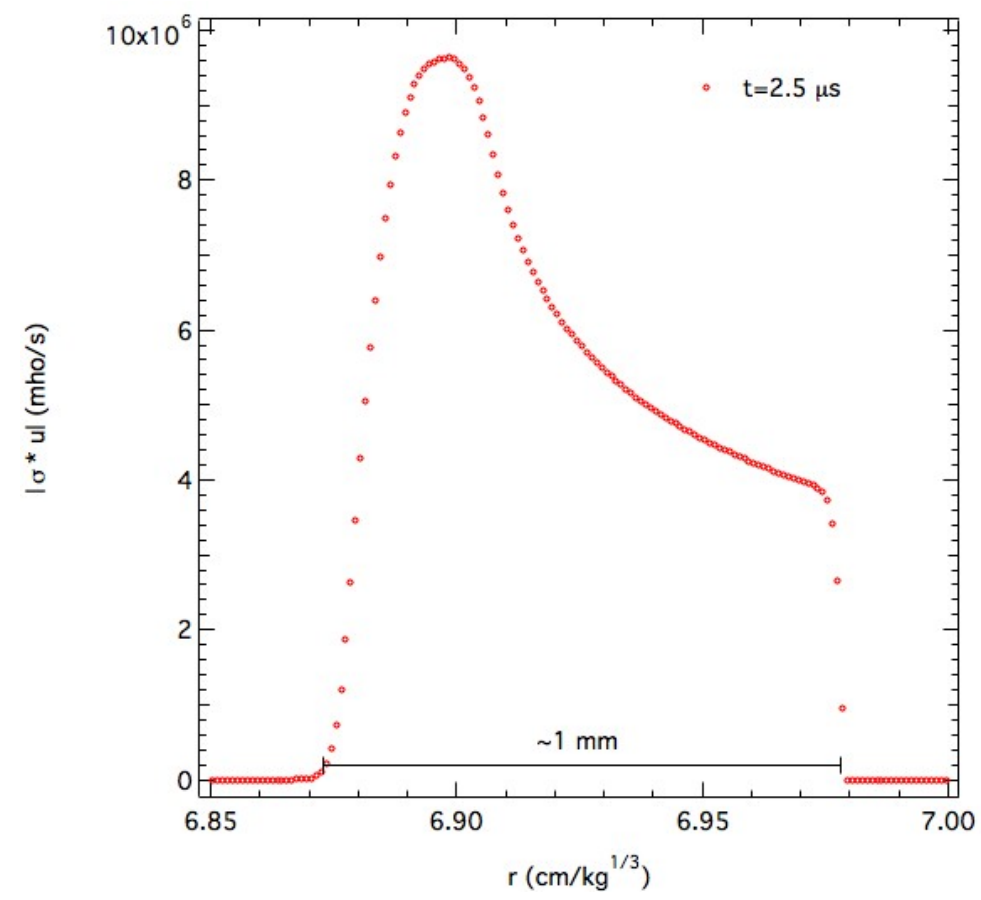

Figure 11. Specific current distribution in the shock-heated air layer of a 1-kg TNT explosion at $2.5 \mu \mathrm{s}$. 


\section{EM Fields from HE Explosions}

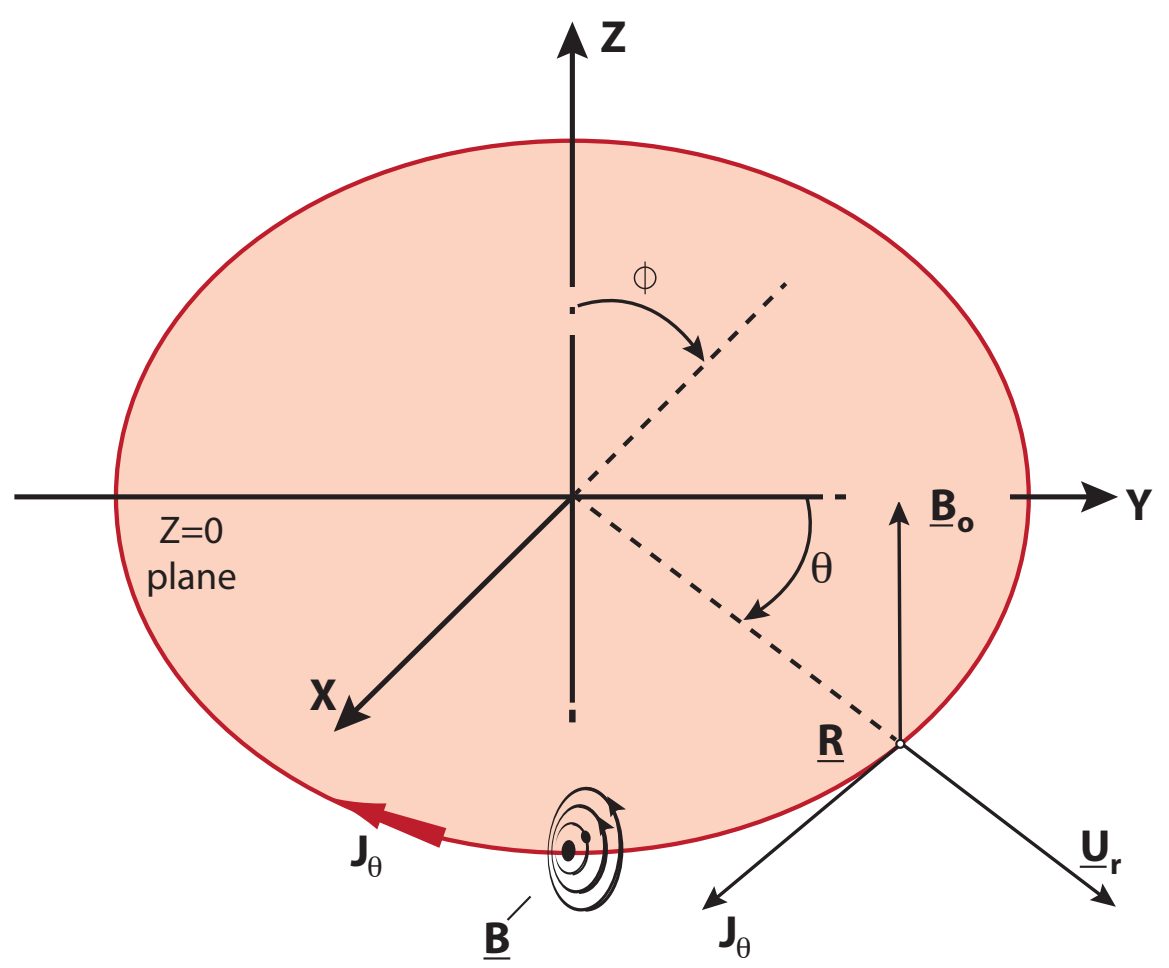

Figure 12. The geometry of the Boronin mechanism for generating a magnetic dipole field. 


\section{A. L. Kuhl}

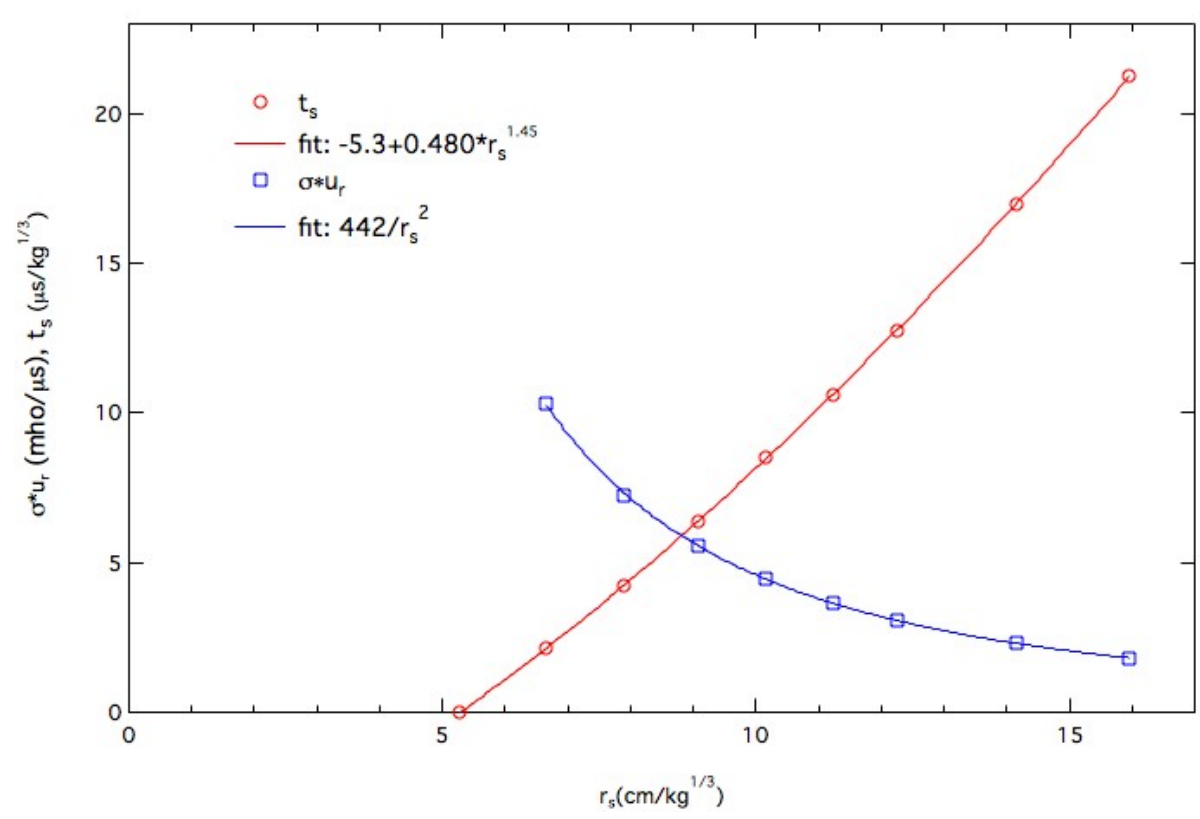

Figure 13. Peak specific current: $\left|\sigma \cdot u_{r}\right|_{s}$ and its scaled arrival time: $t_{s}$ are plotted as a function of scaled radius: $r_{s}$ for a 1-kg spherical TNT charge detonated in air (AMR code simulation). The curve fit implies that the specific current decays as $442 / r_{s}^{2}$.

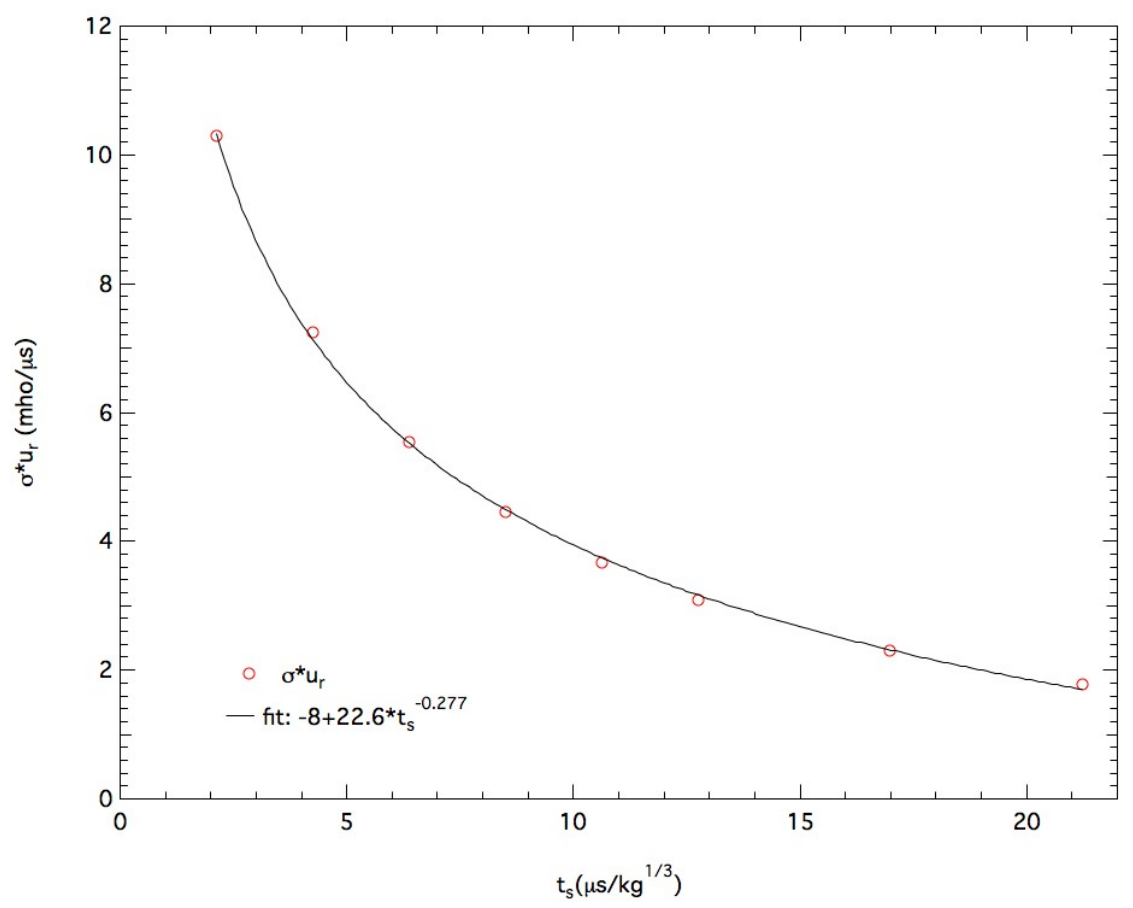

Figure 14. Peak specific current: $\left|\sigma \cdot u_{r}\right|_{s}$ is plotted as a function of its scaled arrival time: $t_{s}$ for a $1-\mathrm{kg}$ spherical TNT charge detonated in air (AMR code simulation). The curve fit implies the specific current decay rate is $-6.26 / t_{s}^{1.277}$. 
(a) TNT-air $(t=50 \mu \mathrm{s})$

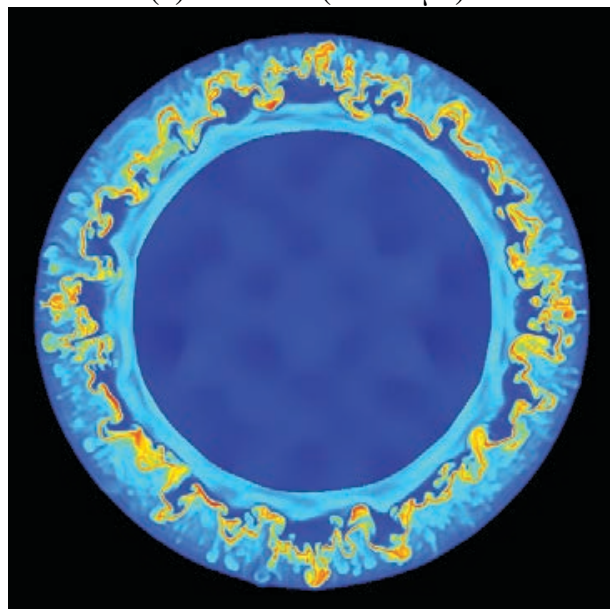

(b) TNT-air $(t=110 \mu \mathrm{s})$

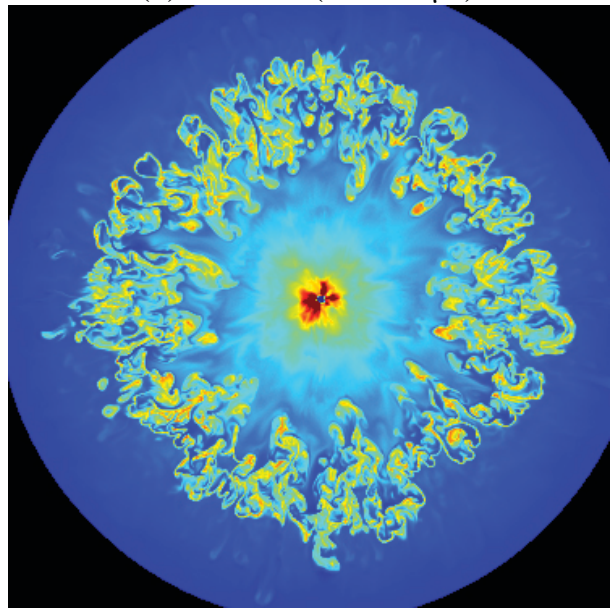

(c) TNT-air $(t=420 \mu \mathrm{s})$

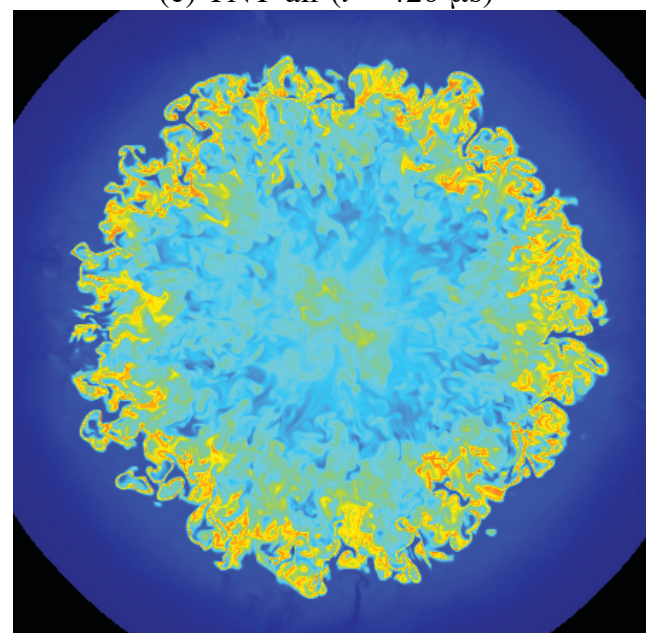

Figure 15. Cross-sectional view of the temperature field of a combustion cloud created by the detonation of a 1-g spherical charge of TNT. Colors denote temperature: red for $3,000<T<$ $4,000 \mathrm{~K}$; yellow for $T=2,000 \mathrm{~K}$; turquoise for $\mathrm{T}=1,000 \mathrm{~K}$; blue for $T=300 \mathrm{~K}$ ). 